2020-05-12

\title{
Fabrication of Polymer Monoliths Within the Confines of Non- transparent 3D-printed Polymer Housings
}

\author{
Noor Abdulhussain \\ University of Amsterdam, n.abdulhussain@uva.nl \\ Suhas H. Nawada \\ University of Amsterdam, S.H.Nawada@uva.nl \\ Sinéad Currivan \\ Technological University Dublin, sinead.currivan@tudublin.ie
}

See next page for additional authors

Follow this and additional works at: https://arrow.tudublin.ie/ittsciart

Part of the Analytical Chemistry Commons, Materials Chemistry Commons, and the Polymer Chemistry Commons

\section{Recommended Citation}

Noor, A., Suhas, N., Currivan, S., Passamonti, M. and Shoenmakers, P. (2020). Fabrication of polymer monoliths within the confines of non-transparent 3D-printed polymer housings. Journal of Chromatography A, vol. 1623, 19 July 2020, 461159. doi:10.1016/j.chroma.2020.461159

This Article is brought to you for free and open access by the School of Science and Computing at ARROW@TU Dublin. It has been accepted for inclusion in Articles by an authorized administrator of ARROW@TU Dublin. For more information, please contact arrow.admin@tudublin.ie, aisling.coyne@tudublin.ie, gerard.connolly@tudublin.ie.

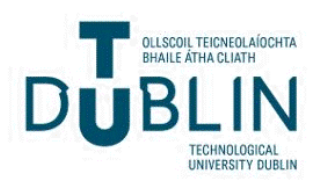




\section{Authors}

Noor Abdulhussain, Suhas H. Nawada, Sinéad Currivan, Marta Passamonti, and Peter J. Schoenmakers

This article is available at ARROW@TU Dublin: https://arrow.tudublin.ie/ittsciart/85 


\title{
Fabrication of polymer monoliths within the confines of non-transparent 3D-printed polymer housings
}

\author{
Noor Abdulhussain a,b,*, Suhas Nawada a,b, Sinéad Currivan ${ }^{c}$, Marta Passamonti ${ }^{\mathrm{a}, \mathrm{b}}$, \\ Peter Schoenmakers ${ }^{\mathrm{a}, \mathrm{b}}$ \\ a Van't Hoff Institute for Molecular Sciences, Science Park, University of Amsterdam 1098 HX Amsterdam, Netherlands \\ ${ }^{\mathrm{b}}$ The Centre for Analytical Sciences Amsterdam (CASA), University of Amsterdam 1098 HX Amsterdam, Netherlands \\ ${ }^{c}$ Centre for Research in Engineering Surface Technology (CREST), Technological University Dublin, FOCAS Research Institute, Camden Row, Dublin 8, Ireland
}

\section{A R T I C L E I N F O}

\section{Article history:}

Received 24 December 2019

Revised 13 April 2020

Accepted 21 April 2020

Available online 12 May 2020

\section{Keywords:}

3D-printing

Additive manufacturing

Monolith

Thermal polymerization

Wall attachment

\begin{abstract}
A B S T R A C T
In the last decade, 3D-printing has emerged as a promising enabling technology in the field of analytical chemistry. Fused-deposition modelling (FDM) is a popular, low-cost and widely accessible technique. In this study, RPLC separations are achieved by in-situ fabrication of porous polymer monoliths, directly within the 3D-printed channels. Thermal polymerization was employed for the fabrication of monolithic columns in optically non-transparent column housings, 3D-printed using two different polypropylene materials. Both acrylate-based and polystyrene-based monoliths were created. Two approaches were used for monolith fabrication, viz. (i) in standard polypropylene (PP) a two-step process was developed, with a radical initiated wall-modification step 2,2'-azobis(2-methylpropionitrile) (AIBN) as the initiator, followed by a polymerization step to generate the monolith; (ii) for glass-reinforced PP (GPP) a silanization step or wall modification preceded the polymerization reaction. The success of wall attachment and the morphology of the monoliths were studied using scanning electron microscopy (SEM), and the permeability of the columns was studied in flow experiments. In both types of housings polystyrene-divinylbenzene (PS-DVB) monoliths were successfully fabricated with good wall attachment. Within the glass-reinforced polypropylene (GPP) printed housing, SEM pictures showed a radially homogenous monolithic structure. The feasibility of performing liquid-chromatographic separations in 3D-printed channels was demonstrated.
\end{abstract}

(C) 2020 Elsevier B.V. All rights reserved.

\section{Introduction}

Microfluidic devices play an important role in many fields, for example in biological, chemical, and engineering applications. Current fabrication is mostly dominated by conventional methods based on soft lithography and moulding, which can produce the necessary channel and feature dimensions. However, with an increasing focus on low-cost fabrication and speed, these approaches face barriers, such as a limitations in geometrical complexity and material choice, that can be overcome by 3D-printing. In recent years, additive manufacturing, or 3D-printing, has gained popularity as a fabrication tool in many fields. It is now commonly viewed as not only a prototyping tool, but as an approach to obtain a final, customized product. The availability and accessibility of 3D-printers make them an attractive alternative for producing customize microfluidic (or "millifluidic") housings [1-5] which can

\footnotetext{
* Corresponding author.

E-mail address: n.abdulhussain@uva.nl (N. Abdulhussain).
}

integrate reagents [4], membranes [4], and structures suitable for chromatographic separations [1,2]

To seamlessly use 3D-printing to produce analytical or chromatographic devices, it is necessary to incorporate stationary phases in-situ in the designed channels. One feasible way to achieve this, in complex geometries, is to synthesize polymer monoliths within the printed channels. A polymer monolith is a single piece of porous polymer, which extends throughout a mould. For liquid-chromatography (LC) applications it is essential that the monolith is well attached to the inner walls of a channel, so as to avoid detrimental mobile-phase flow between the wall and the stationary phase. Polymer monoliths have been investigated extensively over the past 30 years, due to their ease of preparation, affordability, and applicability to many different mould types. Inorganic moulds or housings in which polymeric monoliths have been successfully created include fused silica capillaries and tubes, glass slides [6], and titanium [7,8], while organic housings include cyclic olefin copolymer (COC; chips and tubes) [9-12] and polypropylene (PP; pipette tips and tubes) [13-17] 3D-printed metals, such as 
titanium, provide housing materials with excellent properties $[8,18]$ and the possibility to localize monolith synthesis [19] However, creating such devices is expensive and may be timeconsuming. The modification of printed polymer substrates is challenging, as active groups are generally required on the surface for further reaction, with solvent compatibility also being an issue [2]. This is problematic in 3D- printed materials, where ambiguous composition lists are reported by manufacturers, particularly for inkjet and digital light processing - stereolithography (DLPSLA) printing techniques [2]. Fused-deposition modelling (FDM) provides an attractive alternative, as the ability to create objects with various shapes with low instrument and material cost, no need for chemical post-processing, no resins to cure, and a broad range of materials that can be used to produce prints of high purity. Example materials include polypropylene (PP), polyether ether ketone (PEEK), and graphene [20]. The result is an easily tuneable, cost-effective process. The use of a customized 3D-printed polymer housing for direct attachment of monolithic material in an integrated device is only recently emerging as a field of research [21]. Here, microfluidic devices were 3D-printed using stereolithography with a built-in polymerization window to allow a monolith to be polymerized within the channel. Using a polypropylene filament is attractive, due to the inertness and solvent compatibility of the material. However, these latter aspects also make it highly challenging to chemically attach (monomeric) materials to the surface and relatively challenging to print. The anchoring of a monolith to the housing wall is pivotal for applications in pressuredriven flow. With inorganic housings, this is achieved with silanizing reagents on oxidized surfaces, using one of many different approaches [22]. However, the use of organic housings requires a different approach. If a crosslinking monomer is used as the anchoring molecule $[14,15,17]$, a type-II photoinitiator, such as benzophenone (BP) or 2,2'-azobis(2-methylpropionitrile) (AIBN) is needed to react to the surface.

Methods for the introduction of anchoring groups on the inner walls of polymer housings, often rely on UV-induced immobilization of BP, with a second step to promote polymerization from the surface of the material [14-17]. The mechanism of grafting by BP has been studied in detail by Yang and Rånby and the concept has been applied to a variety of polymer substrates $[10,12,16,17,23]$. BP abstracts a hydrogen from the polymeric surface to initiate polymer grafting [24-27]. The anchoring is performed two steps $[11,12,17]$. The method is effective, but limited to UV-transparent and UV-stable substrates.. Other initiators, such as benzoin methyl ether, and AIBN have been shown to work similarly to type II photoinitiators, such as BP, in abstracting hydrogen atoms from polymeric substrates $[28,29]$. The use of AIBN in graft polymerizations, with varying success, has been documented in the literature $[28,30]$. The effectiveness of AIBN as initiator depends predominantly on the reactivity of the monomer radicals of styrenic, methacrylic, and acrylic monomers. AIBN has been shown to be useful in both single- and multi-step graft-polymerizations to organic substrates. In COC devices, a one-pot monolith synthesis and wall attachment were achieved using AIBN as the initiator with UV irradiation [10]. The monolithic structures were grown in a single step, using a mixture of initiator, monomers and porogens, without an preceding surface modification step. Alternatively, the use of AIBN for secondary polymerizations within polymeric monolithic scaffolds demonstrated the potential for the initiator to be used in thermal grafting reactions on monolithic polymers. Moreover, it has been shown that AIBN can be used to initiate the grafting of polymer monoliths to a pre-existing polymeric scaffold, such as PP housing [15], COC housing [10], or methacrylate-based monolith [31]. Ladner et al. have reported on the use of AIBN in a single-step polymerization of methacrylate monoliths within COC housings [10]. They stipulated that AIBN reacted like a type II pho- toinitiator and they demonstrated that it could be used in a singlestep, one-pot synthesis of methacrylate monoliths in COC using UV initiation. In a thermal approach, Currivan et al. used AIBN to initiate the polymerization of a secondary monolith within a sacrificial monolithic scaffold [31]. Scanning electron microscopy (SEM) images showed the growth of new polymer on the scaffold. This demonstrated that AIBN can be used to initiate surface localized thermal polymerization, as well as polymerization in solution.

In this work, polypropylene (PP) filaments were used to fabricate column housings by FDM. Two approaches were investigated. Firstly a two-step modification reaction was explored for a standard polypropylene filament, using a short reaction time for AIBN and the crosslinker EDMA to provide anchor attachment sites, which was followed by a second polymerization step to generate the monolith using thermally initiated polymerization. Secondly, for a glass-reinforced polypropylene filament, a wall modification step for glass fibres was introduced, followed by a one-pot approach for thermal monolith fabrication.

\section{Materials and methods}

\subsection{Materials}

Azobisisobutyronitrile (AIBN, 98\%), ethylene dimethacrylate (EDMA), butyl methacrylate (BuMA, 99\%), uracil, phenol and ethylbenzene were purchased from Sigma-Aldrich (Darmstadt, Germany). Styrene (S, > 99.5\%), divinylbenzene (DVB, 80\%), 3(trimethoxysilyl)propyl methacrylate ( $\gamma$-MAPS, 98\%), n-decanol (99\%), sodium hydroxide $(\mathrm{NaOH})$, methanol $(\mathrm{MeOH}$, anhydrous, 99.8\%), acetonitrile (ACN) and non-stabilized tetrahydrofuran (THF, HPLC-S grade) were obtained from Biosolve (Valkenswaard, The Netherlands). 1,4-butanediol was obtained from Merck (Darmstadt, Germany).1-propanol (for analysis, 99.5\%) was obtained from Thermofisher Acros Organics (Landsmeer, The Netherlands).

\subsection{Instrumentation}

The modification of the PP housing and the polymerization were carried out using a Julabo water bath (900F, Julabo, Seelbach, Germany). The chromatographic separations were performed on an Agilent Technologies 1100 series system (Agilent, Waldbronn, Germany). For solvent delivery, a capillary pump (G1376A) was used. Other system components included an autosampler (G1313A) and a diode-array detector (G7117B). The morphological and topographical characterization of fabricated monoliths, was performed by scanning electron microscopy (FEI Verios 460, Thermo Fisher Scientific, Eindhoven, The Netherlands) with an Everhart-Thornley detector (EDT). The samples were sputter-coated with gold using an EM ACE600 Double sputter coater (Leica Microsystems, Amsterdam, The Netherlands).

\subsection{Printing of PP housings}

To test the wall attachment, permeability and chromatographic performance of the EDMA monoliths in 3D-printed substrates, a number of column housings containing 2-mm internal-diameter (ID) channels of $45 \mathrm{~mm}$ length, were designed and printed. As seen in Fig. 1A, fitting slots were added for 10-32 fittings to be tapped at the ends of the column. Computer aided design (CAD) files were produced using Autodesk Inventor (San Rafael, CA, USA).

Two filaments were tested, viz. a standard polypropylene (PP) filament (Gizmodorks, Temple City, CA, USA) and a glass-reinforced polypropylene (GPP) (Owens Corning, Toledo, OH, USA) containing 30\% glass fibres (by volume). For the standard PP filament, Ultimaker Cura 3.2.1 (https://ultimaker.com/software/ultimaker-cura) 
Table 1

Print settings used for the standard (PP) and glass-reinforced polypropylene (GPP) filaments

\begin{tabular}{lll}
\hline & Standard Polypropylene $(\mathrm{PP})$ & Glass-Reinforced Polypropylene (GPP) \\
\hline Printer Nozzle Diameter $(\mathrm{mm})$ & 0.35 & 0.6 \\
Layer Thickness $(\mathrm{mm})$ & 0.1 & 0.1 \\
Extruder Temperature $\left({ }^{\circ} \mathrm{C}\right)$ & 240 & 245 \\
Heated-Bed Temperature $\left({ }^{\circ} \mathrm{C}\right)$ & 80 & 90 \\
Chamber Temperature $\left({ }^{\circ} \mathrm{C}\right)$ & $\mathrm{NA}$ & 75 \\
Print Speed $(\mathrm{mm} / \mathrm{s})$ & 20 & 30 \\
Material Flow & $100 \%$ & $110 \%$ \\
Infill Pattern & Cross-Lay & Cross-Lay \\
Supporting Raft & Yes & No \\
\hline
\end{tabular}

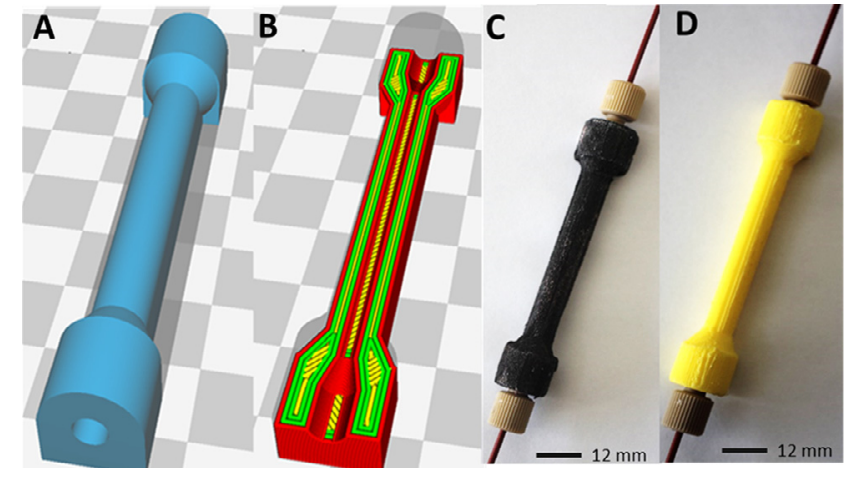

Fig. 1. (a) CAD model of the printed housing with a $2 \mathrm{~mm}$ ID $\times 45 \mathrm{~mm}$ length channel and (b) travel path of the extruder showing the outer casing (red), wall layers (green) and infill (yellow) regions. (c) printed housing of glass-reinforced polypropylene (GPP) and (d) printed housing of standard polypropylene (PP)

was used as the slicing software and the housing was printed using a Felix Pro 2 FDM printer (FelixPrinters, IJsselstein, The Netherlands). For the glass-reinforced PP filament, an FDM printer with a heated-chamber (Intamsys Funmat HT, Intamsys, Ostfildern, Germany) was used to minimize warping and the formation of internal pores within the housings. The CAD files were sliced using the software associated with the printer (Intamsuite 3.4.0, Intamsys).

The full printer settings are shown in Table 1. Both models were printed with a layer thickness of $0.1 \mathrm{~mm}, 100 \%$ infill and with a cross-lay pattern (seen in the diagonal yellow lines in Fig 1b), where orthogonal diagonal lines are used in alternating layers. In this way, the voids introduced during printing of a layer would not be adjacent and parallel to the voids produced by the previous layers. This was doneto ensure water-tightness of the housings and to reduce the number of internal pores.

\subsection{Preparation of monolithic columns}

The printed housing was subjected to two types of modifications. In the first instance, a wall-localized layer of EDMA was formed for subsequent monolith attachment, as described previously elsewhere [15]. In the second instance, a monolith was formed by direct polymerization, in the absence of a linkerfabrication step [10].

\subsubsection{Modification of PP housing for monolith attachment}

In the first approach (column numbers starting with PP1 and PP2 see Table 2) the substrate was washed with water, then with methanol. A solution of 15\% EDMA and 5\% AIBN (by volume) was prepared in methanol. The solution was degassed by nitrogen sparging and flushed into the PP housing, which was sealed and placed in a water bath at $70^{\circ} \mathrm{C}$ during a reaction time of $30 \mathrm{~min}$. Following the reaction, the housings were flushed with methanol, to remove any unreacted components. The printed column was then prepared for the addition of monolith.
Table. 2

Fabricated channels with the two 3D-printed materials: standard and glass reinforced polypropylene (PP and GPP) and monolith fabrication method using ethylene dimethacrylate (EDMA), butyl methacrylate (BUMA) and/or polystyrene divinyl benzene (PS-DVB)

\begin{tabular}{llll}
\hline Column & Material & Method & Monolith type \\
\hline PP1-70 & PP & Short polymerization $\left(70{ }^{\circ} \mathrm{C}\right)$ & EDMA \\
PP1-50 & PP & Short polymerization $\left(50^{\circ} \mathrm{C}\right)$ & EDMA \\
PP1-90 & PP & Short polymerization $\left(90^{\circ} \mathrm{C}\right)$ & EDMA \\
PP2-BE & PP & Two-step approach & BUMA-EDMA \\
PP2-S & PP & Two-step approach & PS-DVB \\
GPP & GPP & Two-step approach & PS-DVB \\
\hline
\end{tabular}

\subsubsection{Wall modification for glass reinforced $P P$}

The reinforced black GPP filament consists of $30 \%$ glass fibres, $68 \%$ PP, and $2 \%$ additives. The pre-treatment and silanization of the glass fibres were carried out as reported [22]. The channels were rinsed with acetone and then with water. After several rinsing steps the channel was filled with an aqueous sodium-hydroxide solution $(0.2 \mathrm{M})$, followed by water (to neutral $\mathrm{pH}$ ), and then with a hydrochloric acid solution $(0.2 \mathrm{M})$. In each of these steps, both ends of the channel were sealed for 30 minutes. After washing with water, ethanol and toluene, the channel was then filled with a solution of $20 \%(\mathrm{v} / \mathrm{v}) 3$-(trimethoxysilyl)propyl methacrylate $(\gamma$ MAPS) in ethanol ( $\mathrm{pH}$ value of approximately 5 , adjusted using acetic acid). The channel was sealed and left for 90 minutes at room temperature, after which it was rinsed with acetone, dried with nitrogen gas, and left overnight. The next day the channel was used in the fabrication of monoliths.

\subsection{Fabrication of monolithic columns}

Two different types of monoliths were fabricated using the 3Dprinted housings. Methacrylate based monoliths were fabricated in the PP housings to study the wall-attachment. PS-DVB monoliths for reversed-phase separations were prepared in GPP housings, as well as in in PP, to allow for a comparison of the morphology obtained in the two materials.

\subsubsection{Fabrication of monoliths}

For fabrication of the BuMA-EDMA monoliths a pre-cursor solution containing BuMA (24 wt\%), EDMA (16 wt\%), 1-propanol (34 wt\%), 1,4 butanediol (26 wt\%) was prepared, with 1\% AIBN (with respect to total monomers present). The solution was degassed using nitrogen sparging for $10 \mathrm{~min}$, before it was brought into the printed housings. Polymerization was achieved using thermal initiation at $70^{\circ} \mathrm{C}$ for $20 \mathrm{~h}$ in a water bath. Following polymerization the channel was connected to an HPLC pump and flushed with $\mathrm{ACN}$ for $1 \mathrm{~h}$.

PS-DVB monoliths were prepared using a solution of styrene (20 wt\%), DVB (20 wt\%), 1-Decanol (52 wt\%), and THF (8 wt\%), with $1 \%$ AIBN (with respect to total monomers present). The remainder 
of the procedure was identical to that used for the BuMA-EDMA monoliths.

\subsection{Monolith characterization}

The monoliths were characterized using chromatographic methods. Back-pressure curves were created for the PS-DVB monoliths in the GPP housings, for water, methanol, acetonitrile and THF with flow rates ranging from $5 \mu \mathrm{L} / \mathrm{min}$ to $500 \mu \mathrm{L} / \mathrm{min}$. The permeability $\left(K_{f}\right)$ of the monolithic columns was measured as described by Darcy's equation (Eq. 1)[32]

$K_{f}=\frac{F_{m} \eta L}{\Delta P \pi r^{2}}$

Where $F_{m}$ is the flow rate $\left(\mathrm{m}^{3} / \mathrm{s}\right), \eta$ is the viscosity $(\mathrm{kg} / \mathrm{m} . \mathrm{s}), L$ is the column length $(\mathrm{m}), r$ is the channel diameter $(\mathrm{m})$, and $\Delta \mathrm{P}$ is the back pressure $(\mathrm{Pa})$.

The swelling propensity (SP) can be used to elucidate the behaviour of the monolithic stationary phase for a variety of solvents and across a solvent gradient. It can also be used to investigate the quality of the monoliths when compared with different solvents. The measurement is based on a gradient of water and organic solvents (Eq. 2)

$S P=\frac{K_{f, \mathrm{~W}}-K_{f, \mathrm{ORG}}}{K_{f, \mathrm{~W}}}$

With an increase in the swelling of the monolith, an increase in pressure and, therefore, an increase in SP is to be expected

\subsection{Separations}

On PS-DVB monolithic columns reversed-phase separations were performed. A mixture of uracil $(0.25 \mathrm{mg} / \mathrm{ml})$, phenol (1 $\mathrm{mg} / \mathrm{ml}$ ) and ethylbenzene $(2 \mathrm{mg} / \mathrm{ml})$ was injected under gradient conditions to evaluate the chromatographic performance of the monolithic material. The gradient used ran from 5 to $60 \%(\mathrm{v} / \mathrm{v})$ of $\mathrm{ACN}$ in water in $2 \mathrm{~min}$, was held at $60 \% \mathrm{ACN}(\mathrm{v} / \mathrm{v})$ for $2 \mathrm{~min}$, and then ramped up to $80 \%$ in 2 min ACN. The flow rate was 400 $\mu \mathrm{L} / \mathrm{min}$ and a wavelength of $210 \mathrm{~nm}$ was set for detection. The injected volume for the samples was $5 \mu \mathrm{L}$.

\section{Results and discussion}

\subsection{Fabrication of monolith}

\subsubsection{PP-housed monoliths}

For creating the EDMA stationary phases, two approaches were tested, one with wall modification to develop a non-porous inner wall, and one without wall modification. The presence of the EDMA had two benefits, i.e. 1) to introduce a vinyl linker, to act as an anchor point for the fabrication of polymer monoliths, and 2) to act as a sealant, along the inner wall of the printed housing, as in the absence of a porogen a non-porous layer would be expected to form. Following the fabrication and washing of the monolithic columns, characterization was performed using SEM. Grafted layers produced using BP (and UV irradiation), which are usually employed for wall modifications in polymer column housings, result in layers varying in thickness between $10 \mathrm{~nm}$ and $5 \mu \mathrm{m}$ [21,31]. To emulate a similar process using thermal initiation, two parameters can be varied, viz. the temperature and the time allowed for the polymerization. Thermal decomposition of the initiating molecule can be influenced by adjusting the temperature. In case of a short polymerization, the reaction time was set for $30 \mathrm{~min}$ and three different temperatures were tested, i.e. $50^{\circ} \mathrm{C}, 70^{\circ} \mathrm{C}$ and $90^{\circ} \mathrm{C}$. A list of fabricated columns is shown in Table 2 (columns PP1). Following the fabrication and washing of the monolithic columns, characterization was performed using SEM. With polymerization at $50^{\circ} \mathrm{C}$ no layer was formed. More surprisingly, this was also the case at $90^{\circ} \mathrm{C}$. For short polymerizations the solutions were prepared in methanol (see method 2.4.1). At a temperature of $90^{\circ} \mathrm{C}$ the solvent evaporates too quickly, resulting in an unsuccessful polymerization.

SEM micrographs are shown in Fig. 2; a cross section of column PP1-70 after 30 min polymerization time at $70^{\circ} \mathrm{C}$ (step 1) is shown in Fig. 2A, with a magnified section of Fig .2 A shown in Fig. 2 B. This resulted in a thin layer of homopolymer, which was observed along the inner walls of the PP inner surface. The formed layer appears to be in the order of 2 to $5 \mu \mathrm{m}$ thick. These values are in the same range as observed within the literature $[24,33]$.

The EDMA layer ensured good wall attachment of the monolith upon subsequent polymerization, as confirmed by the SEM micrographs shown in Fig. 2 (C and D). For comparison, SEM micropgraphs of a monolith with poor wall attachment are shown in the supplementary information, Fig. S1. The micrographs suggest wall attachment, which is aided by the irregular wall geometry of the 3D-printed housing (see Fig. 2C). The monolith is seen to bepresent in the crevices of the housing, with no voids between the wall and the monolith. In some micrographs, monolith is not seen to be present near parts of the wall. However, as monolith appears to be attached to the wall deeper in the sample, the observed void spaces are likely due to the slicing of cross sections for SEM, where the monolith may locally be torn from the wall. The presence of a continuous stationary phase can be confirmed by HPLC separations (vide infra). The monolith showed the expected globular structure, with no discernible evidence of an undesirable radial gradient of the monolith density from the wall to the centre of the monolith (see Fig. S2 of the supporting material for an analysis of the SEM data). Density gradients are usually expected in larger bore housings, as a result of thermal gradients during polymerization [34]. Such gradients would result in a gradual shift in globule size (and thus pore size) across the conduit. The size of the micropore of the monoliths shown in Fig. 2D had a size of the order of $1.1 \mu \mathrm{m}$.

In the absence of a successful surface modification for columns PP1 to PP2, no wall attachment was observed as shown in Fig. $\mathrm{S} 1$ of the supporting material. The addition of EDMA provides a dense network of highly cross-linked homopolymer. A great number of vinyl groups are present in the mixture for polymer attachment, with few - if any - reactive groups present on the PP housing. Thus, no wall attachment is achieved if no anchoring layer is present. For further monoliths the two-step approach was used.

\subsubsection{GPP-housed monoliths}

Fig 2. E and F shows the SEM of a cross section obtained from the GPP housing (GPP). Monoliths are seen to be formed in the GPP housing. The GPP housing in the micrographs shown a rough structure, due to glass fibres in the material (see Fig. S4 in the supporting material). The monolith was attached to the wall, although in some regions (highlighted in Fig. 2E) it had been disrupted during slicing of the printed piece. A magnified section showing the wall attachment in GPP can be seen in Fig. 2F. In GPP the silanol groups, present due to the incorporation of glass, can provide additional anchoring support for the newly formed monolithic material. A silanization step was performed to chemically attach vinyl groups to the surface. These groups can participate in the polymerization reaction to ensure wall attachment.

\subsection{Comparison of Standard PP and Glass-reinforced PP}

In addition to studying wall attachment, SEM was also used to characterize the morphology and topography of the fabricated monoliths. This is illustrated in Fig. 3. To compare the morphology 


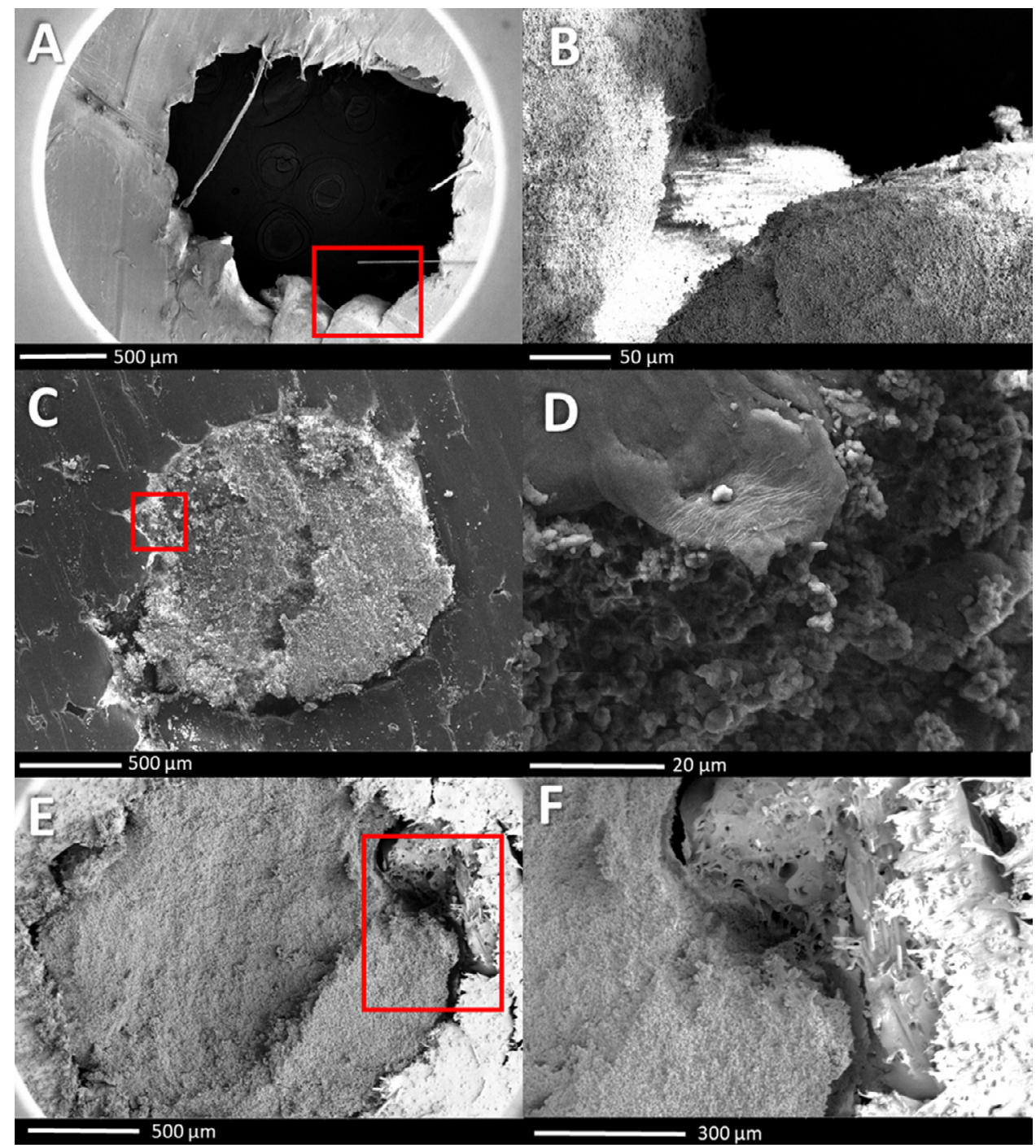

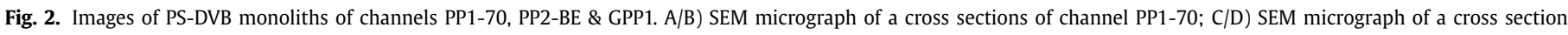
of channel PP2-BE, E/F) SEM micrograph of a section of the channel GPP.

between monoliths created in PP and GPP housings, PS-DVB monoliths were fabricated in both materials, according to the method described in Section 2.5.2. Figs. 3A (PP) and 3B (GPP), show images of a cross section through the entire column. The pictures reveal that significantly different structures are obtained. It can be clearly seen in the enlarged sections shown as Figs. 3C and 3D that the monolith structure in the PP channel (Fig. 3C) has larger globules (ranging from approximately 0.7 to $3.8 \mu \mathrm{m}$ ) and exhibits significant inhomogeneity across the column conduit (see Fig. S3 of the supporting material for an analysis of the SEM data). In contrast, the dense monolith structure in GPP1 appears homogenous and no gradient is visible. The preparation of large-volume, homogeneous monolithic columns is dependent on the composition of the polymerization mixture, the temperature of the water bath, the shape of the channel, the thickness and geometry of the housing, and the heat transfer during the polymerization [35]. The difference in the monolith structure between the two different housing materials is likely due to the heat conductivity of the material (kT). For the standard PP material $\mathrm{kT}$ is expected to fall in the range of 0.17 to $0.22 \mathrm{~W} \cdot \mathrm{m}^{-1} \cdot \mathrm{K}^{-1}$ [36]. The GPP material contains glass fibres, causing an increase in the thermal conductivity to approximately $k_{T}=1 \mathrm{~W} \cdot \mathrm{m}^{-1} \cdot \mathrm{K}^{-1}$. This may imply that the radial temperature profiles of the channels in the two different housings are signif- icantly different during (a) the initial heating phase in the water bath and (b) the exothermic reaction producing the monolithic stationary phase. The GPP housing may allow improved temperature control and faster heat transfer to dissipate the excess heat during the polymerization. As a result, homogenous monolithic structures were formed as shown in Fig. 3 C. Moreover, the monolithic structure in GPP is seen to be denser than that in the standard PP. This would result in a lower permeability and increased backpressure.

However, FDM printing technology has some drawbacks regarding the 3D-printed channels. To understand the functional capabilities and limitations of FDM manufactured components, control over their micro-structural properties (i.e. of the extruded lines of PP and GPP) is crucial. For example, voids introduced during the production phase greatly affect the strength of the printed object. For the standard PP channels, such voids meant that a backpressure exceeding 30 bar led to approximately $30-50 \%$ loss of flow through the column housing and through the connector ports. This prohibits a meaningful analysis of the backpressure and permeability of the monolith created in the standard PP-filament housing. This issue can be partly ameliorated by changing printing parameters, such as the filament flow and the orientation ("lay") of the printed lines [37]. Optimizing the printing process results in channels that are adequate for the low pressures generally used 


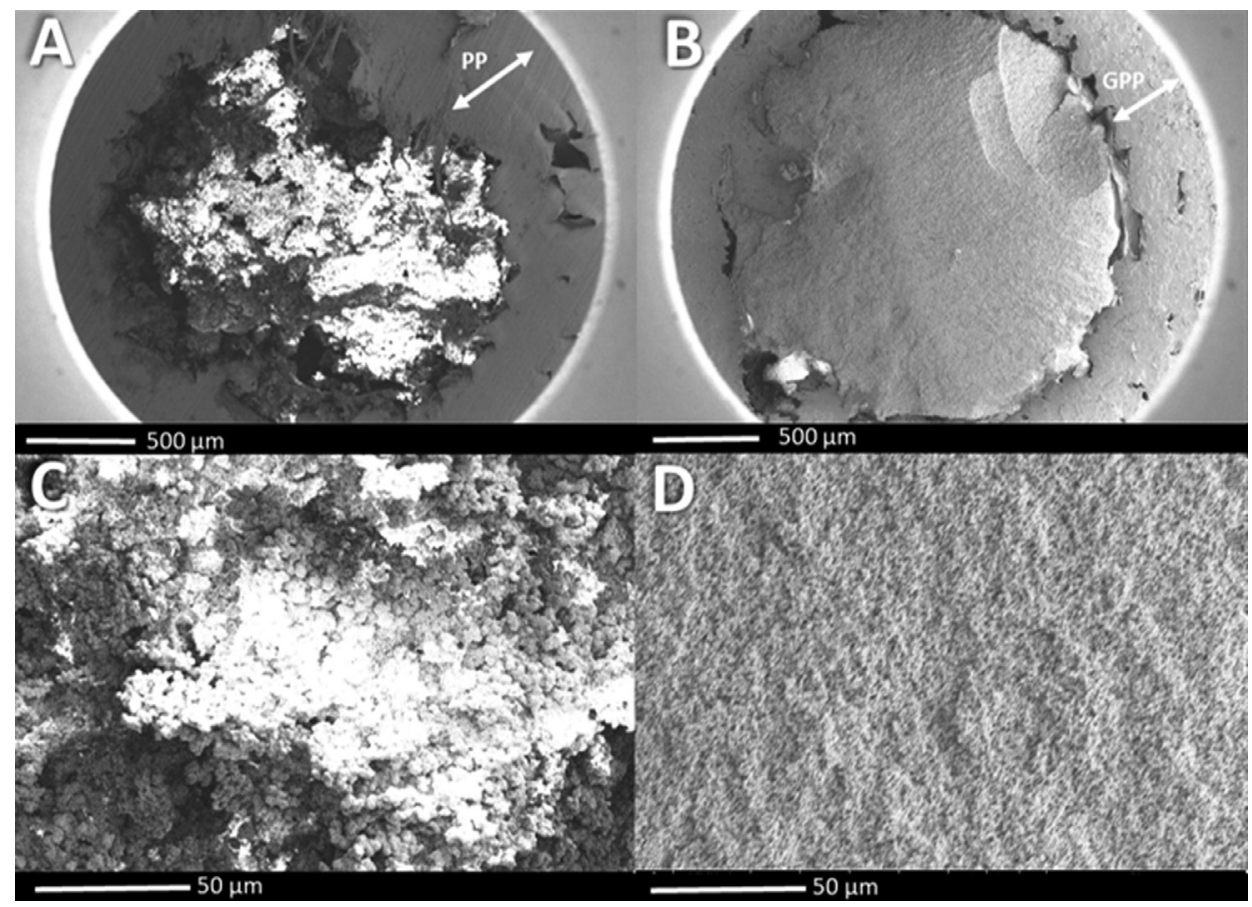

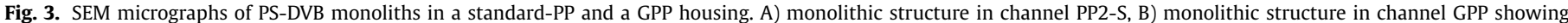
a homogenous structure, C) monolithic globules obtained in PP channels, D) monolithic globules in GPP channel.

in microfluidic applications, but won't suffice for pressures used in HPLC. Low pressure applications with regular FDM printers have limits such as warping, due to crystallinity of the PP material. This can be overcome by using FDM printer with a heated chamber to better control the change in crystallinity during the print to make more pressure-resistant columns. GPP channels were printed using a FDM printer with a heated-chamber, which can facilitate better inter-layer adhesion. Therefore, permeability and back-pressure studies were performed for the GPP-housed monoliths (column GPP). Despite the use of a stronger filament and a more robust printing method, voids within the printed pieces were not completely eliminated. To effectively use the homogenous and wellattached monoliths produced inside the GPP housing for highpressure applications, several aspects of the printing process need to be further optimized. These include the inter-layer adhesion of the GPP filament, filament flow speeds, and extrusion-nozzle diameters.

\subsection{Characterization of monoliths in GPP housings}

To evaluate the permeability and mechanical stability of the monolithic stationary phase in contact with organic solvents, the pressure drop across the housing was measured with various solvents (water, methanol, ACN and THF) across a wide range of flow rates. Each measurement was performed in triplicate and preceded by a 15-min equilibration period. The averages of the observed pressure were plotted against the flow rate (Fig. 4). The backpressure is seen to vary approximately linearly with the flow rate for each solvent tested (regression coefficient $\mathrm{R}>0.998$ for all solvents). For the highest flow rate of $500 \mu \mathrm{L} / \mathrm{min}$ in water, a back pressure of 38 bar was observed. A lower pressure drop was observed for methanol (36 bar at $500 \mu \mathrm{L} / \mathrm{min}$ ) and acetonitrile (30 bar at $500 \mu \mathrm{L} / \mathrm{min}$ ). The low standard deviations across the three repeated runs indicates good mechanical stability of both the polymer monolith, as well as the printed structure at the tested pressures (table S-1 in the supporting information).

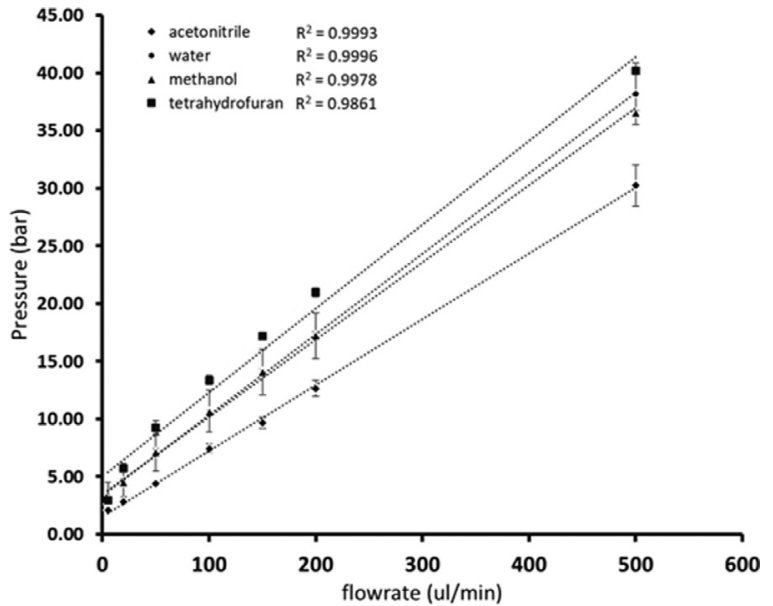

Fig. 4. Measured pressure drop vs. flow rate with various solvents for a PS-DVB monolith in a GPP housing (Column GPP), $n=3$

The equivalent particle diameter for the monolithic stationary phase, as derived from the permeability by Darcy's Law [32], is 1.8 $\mu \mathrm{m}$ for water. For a monolith with a porous structure the pressure drop across a column at a given flow rate will only dependent on the viscosity of the solvent. However, it is well-known that polymer monolithic stationary phases may experience a certain degree of swelling. The permeability with water is just $86 \%$ higher than with acetonitrile, whereas the viscosity is $157 \%$ higher. This suggests that some swelling of the stationary phase, narrowing of the flow-through pores and, possibly, restriction of the accessible pore volume occurs when using acetonitrile. The swelling propensity for the monolith in the different solvents (SP, see Eqn. 2) is listed in Table 3. The degree of swelling is slightly less for THF and methanol than for acetonitrile, but similar for all three solvents. 
Table. 3

Viscosity at $20^{\circ} \mathrm{C}$ of solvents and permeability at the highest flow rates tested ( $\left.500 \mu \mathrm{L} / \mathrm{min}\right)$ for channel GPP1

\begin{tabular}{lllll}
\hline Solvent & viscosity $\eta(\mathrm{kg} /(\mathrm{ms}))$ & Pressure at $500 \mu \mathrm{L} / \mathrm{min}(\mathrm{MPa})$ & Permeability $K_{f}\left(\mathrm{~m}^{2}\right)$ & $\mathrm{SP}(\mathrm{Eq} .2)$ \\
\hline Water & $1.00 \cdot 10^{-3}$ & 3.8 & $4.15 \cdot 10^{-14}$ & 0.00 \\
Acetonitrile & $3.89 \cdot 10^{-4}$ & 3.0 & $2.23 \cdot 10^{-14}$ & 0.46 \\
Methanol & $5.94 \cdot 10^{-4}$ & 3.6 & $2.61 \cdot 10^{-14}$ & 0.37 \\
Tetrahydrofuran & $4.80 \cdot 10^{-4}$ & 4.0 & $2.36 \cdot 10^{-14}$ & 0.43 \\
\hline
\end{tabular}

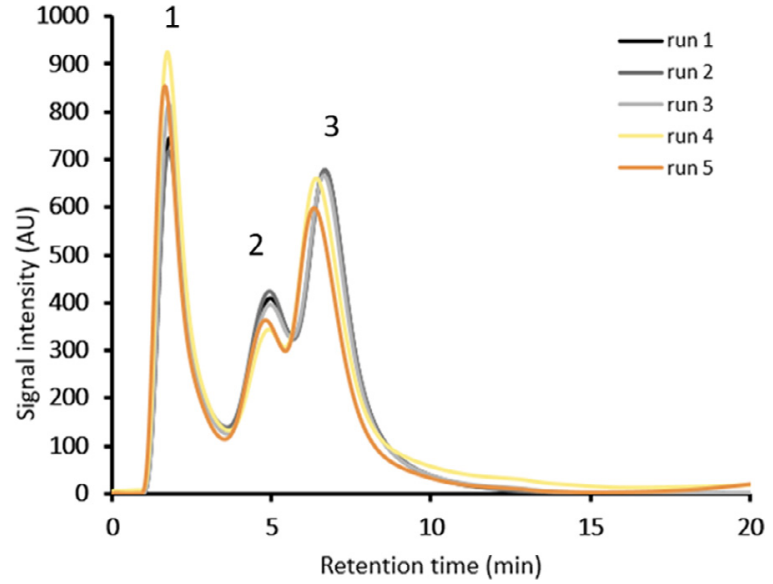

Fig. 5. Separation of small molecules on channel GPP1 using a gradient used ran from 5 to $60 \%(\mathrm{v} / \mathrm{v})$ of $\mathrm{ACN}$ in water in $2 \mathrm{~min}$, was held at $60 \% \mathrm{ACN}(\mathrm{v} / \mathrm{v})$ for 2 min, and then ramped up to $80 \%$ in 2 min ACN. Analytes: (1) Uracil, (2) phenol, (3) ethylbenzene; flow rate $400 \mu \mathrm{L} / \mathrm{min}$. Separation performed at room temperature with UV detection at $210 \mathrm{~nm}$.

The backpressure values shown in Table 3 are especially pertinent during gradient separations in RPLC, where the swelling of the monolith and the shrinking of the pores may increase during the run, as a solvent gradient is applied to the column. Swelling may lead to changes in pore size, plate number, binding capacity, etc [38]. Such effects must be taken into consideration when performing or developing gradient separations.

Despite the swelling mentioned above, it is worth noting that the monolith and the printed housing did not experience any discernible deterioration during the tests with strong organic solvents, such as THF or acetonitrile. The solvent resistance of polypropylene combined with the stiffness and tensile strength provided by the glass fibres, make for a favourable housing material that is compatible with common HPLC solvents.

\subsection{Chromatographic separation on a monolith in PP housing}

To demonstrate the feasibility of performing chromatographic separations and repeatability on columns formed within 3Dprinted polymer housings, a test mixture of uracil (void marker), phenol and ethylbenzene was injected on a PS-DVB monolith (column GPP). At a flow rate of $400 \mu \mathrm{L} / \mathrm{min}$ uracil (peak \#1 in Fig. 5, retention time $1.8 \mathrm{~min}$ ), phenol (peak\#2, $5.0 \mathrm{~min}$ ) and ethylbenzene (peak\#3, $6.6 \mathrm{~min}$ ) could be separated under gradient conditions. No separation occurred under isocratic conditions. The peak of the unretained marker (which traverses the entirety of the column) exhibits some tailing, suggesting that some channelling may occur in the column. The two retained peaks, although not baseline separated, indicate the successful attachment of the monolith to the wall of the printed housing. The retention and shapes of these two peaks also suggest limited migration of the analytes from the monolith into (and out of) voids in the printed filament. The repeatability of the chromatographic separations in the channel was measured by calculation the RSD values of the retention times.
Table 4

RSD values of the retention times of the repeated measurements for all three components.

\begin{tabular}{|c|c|c|c|}
\hline \multicolumn{2}{|c|}{ Number of runs } & \multicolumn{2}{|c|}{ Retention times (min) } \\
\hline & Uracil & Phenol & Ethylbenzene \\
\hline 1 & 1.80 & 5.00 & 6.68 \\
\hline 2 & 1.78 & 5.00 & 6.69 \\
\hline 3 & 1.78 & 4.99 & 6.65 \\
\hline 4 & 1.73 & 4.94 & 6.42 \\
\hline 5 & 1.65 & 4.85 & 6.35 \\
\hline Average & 1.75 & 4.96 & 6.56 \\
\hline RSD\% & 3.0 & 1.2 & 2.2 \\
\hline
\end{tabular}

For all components, the RSD values were below $3 \%$, as shown in Table 4.

The significant band broadening observed in Fig. 5 may have several causes, such as the rudimentary design of the inlet and outlet regions. In the design tested here, no flow distributor incorporated. Instead the inlet ferrule was pressed against a $2-\mathrm{mm}$ ID region of bulk monolith. This inevitably introduces structural inhomogeneities in the inlet and outlet regions of the column. This can be mitigated by modifying the column design and introducing narrower inlet and outlet channels that gradually tapers out to a diameter of $2 \mathrm{~mm}$. Another potential reason are the striations caused by the FDM printing process; as seen in Figs. 2 and 3, the channels do not feature a perfectly circular crosssection. Rather, a series of striations are observed across the channel wall. Improving the shape of the column's cross-section will require the use of narrower printing nozzles. Moreover, the lack of resolution between peaks 2 and 3 may be indicative of the micro/mesoporosity of the monolith, which is known to be poor in polymer-monolithic columns, particularly those fabricated with $20 \mathrm{~h}$ polymerization times $[24,33]$. Moreover, currently column-tocolumn repeatability is still an issue, due to irregularities in the monolithic structures introduced when making the fittings; therefore causing insufficient separations and unreliable retention times. Polymer-monolithic columns are commonly thought to be better suited for the separation of large biomolecules. Separations of large molecules (peptides or proteins) were attempted, but no separation was achieved. This was due to the dense monoliths and narrow flow-through pores. Ultimately, we aim to realize separations with other retention mechanisms for other applications, such as peptides or proteins, by tailoring the morphology of the PS-DVB monoliths. The concentration of THF in the polymerization mixture may be lowered, which will result in larger monolith globules, and thus larger throughpores. We envisage that, depending on the type of application, different types of monoliths can be prepared to realize separations within 3D-printed polymer housings with arbitrary geometries and dimensions.

\section{Conclusion}

3D-printed channels for the separation of small molecules were successfully developed in-house. Two different materials were compared, viz. a regular ("standard") polypropylene (PP) material and glass-reinforced polypropylene (GPP) for printing column 
housings. In both types of housings PS-DVB monoliths were successfully fabricated with good wall attachment. Within the GPP printed housing, SEM micropgraphs showed a radially homogenous monolithic structure. Permeability and back pressure studies were performed with the GPP-housed monoliths. The columns prepared in standard PP showed lower pressure resistance due to and voids in the PP printed housing. The GPP-housed monoliths showed a stable permeability for flow rates up to $500 \mu \mathrm{L} / \mathrm{min}$, although they exhibited some swelling with different organic solvents. A separation was achieved with a mixture of small molecules, although without baseline separation. The housing $(45 \times 2.0 \mathrm{~mm}$ i.d. $)$ was printed using a GPP filament with a fused-deposition modelling (FDM) printer with a heated-chamber. Improving the irregularities in the monolith structure introduced when making the fittings, will allow improved column-to-column repeatability in the future. Using GPP instead of PP allowed us to combine the solvent resistance of polypropylene with the superior pressure resistance and improved water tightness offered by the composite material. Nevertheless, several improvements may still possibly be made to the printing of GPP housing. These include printing with smaller nozzles, to obtain more-regular and more-circular channels, using selective laser sintering to avoid pores and reduce leakage [39], and applying coatings after printing. Surface coatings can provide supplementary and novel surface chemistries, while providing a protective and/or sealing layer. Mireles et al. studied the sealing of 3Dprinted parts with various types of coatings and demonstrated that they could eliminate voids and reinforce bonds between printed layers, so as to prevent leakage and to improve the mechanical strength of the device [40]. Potentially, FDM can be used to produce columns in-house, for a fraction of the cost, with the ability to specify bespoke (on-demand) column dimensions. The method reported here can serve as the template for designing 3D-printed devices with monolithic stationary phases for a wide variety of applications. New insights for further applications can be obtained by extending this work to create monoliths in confined parts of morecomplex printed objects [19] or for applications in solid-phase extraction (SPE) or immobilized-enzyme reactors (IMERs).

\section{Declaration of Competing Interests}

None.

\section{CRediT authorship contribution statement}

Noor Abdulhussain: Methodology, Investigation, Writing - original draft. Suhas Nawada: Methodology, Software, Formal analysis, Writing - review \& editing. Sinéad Currivan: Formal analysis, Writing - review \& editing. Marta Passamonti: Investigation. Peter Schoenmakers: Supervision, Writing - review \& editing.

\section{Acknowledgments}

The STAMP project is funded under Horizon 2020-Excellent Science-European Research Council (ERC), Project 694151. The sole responsibility of this publication lies with the authors. The European Union is not responsible for any use that may be made of the information contained therein.

\section{Supplementary materials}

Supplementary material associated with this article can be found, in the online version, at doi:10.1016/j.chroma.2020.461159.

\section{References}

[1] C. Fee, S. Nawada, S. Dimartino, 3D printed porous media columns with fine control of column packing morphology, J. Chromatogr. A. 1333 (2014) 18-24, doi:10.1016/j.chroma.2014.01.043.
[2] N.P. Macdonald, S.A. Currivan, L. Tedone, B. Paull, Direct production of microstructured surfaces for planar chromatography using 3D printing, Anal Chem. 89 (2017) 2457-2463, doi:10.1021/acs.analchem.6b04546.

[3] F. Li, N.P. Macdonald, R.M. Guijt, M.C. Breadmore, Using printing orientation for tuning fluidic behavior in microfluidic chips made by fused deposition modeling 3D printing, Anal. Chem. 89 (2017) 12805-12811, doi:10.1021/acs.analchem. 7 b03228.

[4] F. Li, P. Smejkal, N.P. Macdonald, R.M. Guijt, M.C. Breadmore, One-step fabrication of a microfluidic device with an integrated membrane and embedded reagents by multimaterial 3D printing, Anal. Chem. 89 (2017) 4701-4707, doi:10.1021/acs.analchem.7b00409.

[5] N.P. Macdonald, J.M. Cabot, P. Smejkal, R.M. Guijt, B. Paull, M.C. Breadmore, Comparing microfluidic performance of three-dimensional (3D) printing platforms, Anal. Chem. 89 (2017) 3858-3866, doi:10.1021/acs.analchem.7b00136.

[6] I. Urbanova, F. Svec, Monolithic polymer layer with gradient of hydrophobicity for separation of peptides using two-dimensional thin layer chromatography and MALDI-TOF-MS detection, J. Sep. Sci. 34 (2011) 2345-2351, doi:10.1002/ jssc.201100202.

[7] E.P. Nesterenko, P.N. Nesterenko, D. Connolly, F. Lacroix, B. Paull, Micro-bore titanium housed polymer monoliths for reversed-phase liquid chromatography of small molecules, J. Chromatogr. A (2010), doi:10.1016/j.chroma.2010.02.007.

[8] V. Gupta, M. Talebi, J. Deverell, S. Sandron, P.N. Nesterenko, B. Heery, F. Thompson, S. Beirne, G.G. Wallace, B. Paull, Analytica chimica acta 3D printed titanium micro-bore columns containing polymer monoliths for reversed-phase liquid chromatography, Anal. Chim. Acta 910 (2016) 84-94, doi:10.1016/j.aca. 2016.01.012.

[9] K. Flook, Y. Agroskin, C. Pohl, Reversed-phase monoliths prepared by UV polymerization of divinylbenzene, J. Sep. Sci. 34 (2011) 2047-2053, doi:10.1002/ jssc.201100196.

[10] Y. Ladner, A. Bruchet, G. Crétier, V. Dugas, J. Randon, K. Faure, New “one-step” method for the simultaneous synthesis and anchoring of organic monolith inside COC microchip channels, Lab Chip 12 (2012) 1680-1685, doi:10.1039/ c21c21211k.

[11] D.A. Mair, T.G. Holden, L.J. Lee, F. Svec, Original paper hydrophilic surface modification of cyclic olefin copolymer microfluidic chips using sequential photografting, (2007) 1088-1093. doi: 10.1002/jssc.200600515.

[12] T.B. Stachowiak, F. Svec, J.M.J. Fre, Patternable protein resistant surfaces for multifunctional microfluidic devices via surface hydrophilization of porous polymer monoliths using photografting, (2006) 5950-5957. doi: $10.1021 / \mathrm{cm} 0617034$.

[13] V. Chunhakorn, P. Ratchathamma, H.J. Whitlow, O. Chienthavorn, Inexpensive simple extraction of trace PAHs from water using PS-DVB monolithic beads, Anal. Methods 10 (2018) 4813-4820, doi:10.1039/c8ay01562g.

[14] M. Iacono, D. Connolly, A. Heise, Polymer brush decorated nanoparticles immobilised on polymer monoliths for enhanced biopolymer elution, RSC Adv. 7 (2017) 19976-19981, doi:10.1039/c7ra02839c.

[15] M. Iacono, D. Connolly, A. Heise, Fabrication of a GMA-co-EDMA monolith in a $2.0 \mathrm{~mm}$ i.d. polypropylene housing, Materials (Basel) 9 (2016) 1-10, doi:10. $3390 /$ ma9040263.

[16] J. Krenkova, F. Foret, Nanoparticle-modified monolithic pipette tips for phosphopeptide enrichment, Anal. Bioanal. Chem. 405 (2013) 2175-2183, doi:10 1007/s00216-012-6358-z.

[17] H. Alwael, D. Connolly, P. Clarke, R. Thompson, B. Twamley, B. O'Connor, B. Paull, Pipette-tip selective extraction of glycoproteins with lectin modified gold nano-particles on a polymer monolithic phase, Analyst 136 (2011) 26192628, doi:10.1039/c1an15137a.

[18] S. Sandron, B. Heery, V. Gupta, D.A. Collins, E.P. Nesterenko, P.N. Nesterenko, M. Talebi, S. Beirne, F. Thompson, G.G. Wallace, D. Brabazon, B. Paull, 3D printed metal columns for capillary liquid chromatography, Analyst 139 (2014) 6343-6347, doi:10.1039/c4an01476f.

[19] M. Passamonti, I.L. Bremer, S.H. Nawada, S.A. Currivan, A.F.G. Gargano, P.J. Schoenmakers, Confinement of monolithic stationary phases in targeted regions of 3D-printed titanium devices using thermal polymerization, Anal. Chem. (2020), doi:10.1021/acs.analchem.9b04298.

[20] H. Guo, R. Lv, S. Bai, Recent advances on 3D printing graphene-based composites, Nano Mater. Sci. 1 (2019) 101-115, doi:10.1016/j.nanoms.2019.03.003.

[21] E.K. Parker, A.V. Nielsen, M.J. Beauchamp, H.M. Almughamsi, J.B. Nielsen, M. Sonker, H. Gong, G.P. Nordin, A.T. Woolley, 3D printed microfluidic devices with immunoaffinity monoliths for extraction of preterm birth biomarkers, Anal. Bioanal. Chem. 411 (2019) 5405-5413, doi:10.1007/s00216-018-1440-9.

[22] J. Courtois, M. Szumski, E. Byström, A. Iwasiewicz, A. Shchukarev, K. Irgum, Erratum: A study of surface modification and anchoring techniques used in the preparation of monolithic microcolumns in fused silica capillaries (Journal of Separation Science (2006) vol. (29) (1) (14-24)), J. Sep. Sci. 29 (2006) 324, doi:10.1002/jssc.200500294.

[23] T.C. Logan, D.S. Clark, T.B. Stachowiak, F. Svec, J.M.J. Fre, Photopatterning enzymes on polymer monoliths in microfluidic devices for steady-state kinetic analysis and spatially separated multi-enzyme reactions polymer monoliths within microfluidic devices has been the observed maximum reaction rate and Michaelis, 79 (2007) 6592-6598. doi: 10.1021/ac070705k.

[24] W. Yang, B. Ranby, Bulk surface photografting process and its applications. I. reactions and kinetics, (n.d.)533-543.

[25] W. T. Yang, B. Ranby, The role of far UV radiation in the photografting process, Polymer Bulletin 37 (1996) 89-96. 
[26] W. T. Yang, B. Ranby, Bulk Surface Photografting Process and Its Application. III. Photolamination of Polymer Films, Journal of Applied Polymer Science 62 (1996) 1723-1732.

[27] W. T. Yang, B. Ranby, Bulk Surface Photografting Process and Its Applications. II. Principal Factors Affecting Surface Photografting, Journal of Applied Polymer Science 62 (1996) 545-555.

[28] L. Tomašek, A. Jukić, Z. Janović, Free radical grafting of methyl methacrylate onto ethylene-propylene amorphous copolymer, Croat. Chem. Acta 82 (2009) 825-832.

[29] M. Uygun, M.A. Tasdelen, Y. Yagci, Influence of type of initiation on thiolene "click" Chemistry, Macromol. Chem. Phys. 211 (2010) 103-110, doi:10.1002/ macp.200900442.

[30] N. -J Huang, D.C. Sundberg, Fundamental studies of grafting reactions in free radical copolymerization. I. A detailed kinetic model for solution polymerization, J. Polym. Sci. Part A Polym. Chem. 33 (1995) 2533-2549, doi:10.1002/pola. 1995.080331502.

[31] S. Currivan, J.M. Macak, P. Jandera, Polymethacrylate monolithic columns for hydrophilic interaction liquid chromatography prepared using a secondary surface polymerization, J. Chromatogr. A. 1402 (2015) 82-93, doi:10.1016/j. chroma.2015.05.016.

[32] R.D. Stanelle, L.C. Sander, R.K. Marcus, Hydrodynamic flow in capillary-channel fiber columns for liquid chromatography, J. Chromatogr. A. 1100 (2005) 68-75, doi:10.1016/j.chroma.2005.09.014.
[33] B. Rånby, W.T. Yang, O. Tretinnikov, Surface photografting of polymer fibers, films and sheets, Nucl. Instruments Methods Phys. Res. Sect. B Beam Interact. Mater. Atoms 151 (1999) 301-305, doi:10.1016/S0168-583X(99)00158-5.

[34] G. Guiochon, Monolithic columns in high-performance liquid chromatography, J. Chromatogr. A. 1168 (2007) 101-168, doi:10.1016/j.chroma.2007.05.090.

[35] C.P. Bisjak, L. Trojer, S.H. Lubbad, W. Wieder, G.K. Bonn, Influence of different polymerisation parameters on the separation efficiency of monolithic poly(phenyl acrylate-co-1,4-phenylene diacrylate) capillary columns, J. Chromatogr. A. 1154 (2007) 269-276, doi:10.1016/j.chroma.2007.03.100.

[36] J.R. Rumble, Handbook of Chemistry and Physics (2017).

[37] G.I.J. Salentijn, P.E. Oomen, M. Grajewski, E. Verpoorte, Fused deposition modeling 3D printing for (Bio)analytical device fabrication: procedures, materials, and applications, Anal. Chem. 89 (2017) 7053-7061, doi:10.1021/acs.analchem. 7 b00828.

[38] M. Nevejans, F. Verzele, swelling propensity (SP factor) of semi-rigid chromatographic packing materials, J. Chromatogr. (1985) 145-150.

[39] I.F. Ituarte, O. Wiikinkoski, A. Jansson, Additive manufacturing of polypropylene: a screening design of experiment using laser-based powder bed fusion, Polymers (Basel) (2018) 10, doi:10.3390/polym10121293.

[40] J. Mireles, A. Adame, D. Espalin, F. Medina, R. Winker, T. Hoppe, B. Zinniel, R. Wicker, Analysis of sealing methods for FDM-fabricated parts, Solid Free. Fabr. (2011) 185-196. 